\title{
Association Between Nonaneurysmal Cortical Subarachnoid Hemorrhage and Intracranial Arterial Stenosis
}

\author{
Jeong Kyu Lee, MD* (D); Dae Wang Jeong, MD* (D; Seong Yeol Ahn, $\mathrm{MD}^{\dagger}$ (D); Jong-Ho Park, MD, PhD ${ }^{*}$ (D) \\ Departments of Neurology ${ }^{\star}$, Neurosurgery ${ }^{\dagger}$, Myongji Hospital, Hanyang University College of Medicine, Goyang, Korea
}

Nonaneurysmal cortical subarachnoid hemorrhage (cSAH) is a rare condition associated with various etiologies. Given the association between carotid artery stenosis and cSAH, we report a case of a patient with hemorrhage associated with significant intracranial arterial stenosis. Furthermore, we discuss angiographic findings to support the relationship between hemodynamic stress and leptomeningeal arterial damage, especially in the watershed region.

J Neurosonol Neuroimag 2021;13(1):18-21

Key Words: Subarachnoid hemorrhage; Intracranial cerebral arterial disease; Hemodynamics; Collateral circulation

Nonaneurysmal cortical subarachnoid hemorrhage (cSAH) is a rare condition associated with bleeding localized in the brain convexity, without involvement of the brain parenchyma or extension into the interhemispheric fissure, basal cistern, or ventricle. cSAH is known to be associated with multiple vascular etiologies, including reversible cerebral vasoconstriction syndrome, cerebral amyloid angiopathy, dural fistula, cerebral venous thrombosis, or high-grade atherosclerotic stenosis. ${ }^{1}$ However, the contribution of highgrade stenosis to the development of cSAH remains unclear. It has been speculated that the acute increase in hemodynamic stress damages the pial arteries in the ipsilesional cortex, which dilates as a compensatory mechanism. Hemodynamic stress-related extracranial internal carotid artery stenosis is the most commonly reported cause of $\mathrm{cSAH}^{1-3}$; however, there is insufficient evidence to support the role of intracranial stenosis in the development of cSAH. Herein, we present a case of a patient with $\mathrm{cSAH}$ associated with middle cerebral artery (MCA) stenosis and discuss angiographic and perfusion findings to support the plausible mechanism of pial arterial damage.
Received: April 28, 2021

Revised: June 4, 2021

Accepted: June 4, 2021

Correspondence:

Jong-Ho Park, MD, PhD

Department of Neurology, Myongji Hospital, Hanyang University College of Medicine, 55 Hwasu-ro 14beon-gil, Deogyang-gu, Goyang 10475, Korea Tel: $+82-31-810-5460$

Fax: +82-31-969-0500

E-mail: neurotector.jhp@ gmail.com

\section{CASE}

A 55-year-old man, a former 30 pack-year smoker, had been diagnosed with silent cerebral infarction 4 years ago and was treated with aspirin $100 \mathrm{mg} /$ day. The patient who overworked as a bus driver experienced a sudden onset of thunderclap headache with nausea and vomiting lasting for 2 days. He had no history of hypertension and head trauma or use of vasoactive substances, including cannabis, selective serotonin-reuptake inhibitors, or sympathomimetic drugs. Upon arrival, his blood pressure was 161/95 mmHg. Neurological examination revealed no significant findings. Initial non-contrast brain computed tomography (CT) showed faint hyperattenuation along the left frontoparietal sulci (Supplementary Fig. 1). Brain diffusion-weighted imaging demonstrated a small acute infarct in the left temporal lobe (Supplementary Fig. 2), whereas gradient-echo imaging revealed hemorrhage along the left frontoparietal cortical sulci and bilateral sylvian fissures (Fig. 1). Time-of-flight (TOF) brain magnetic resonance (MR) angiography showed an irregular tight stenosis along the left MCA with occlusion of the 
right MCA, whereas the anterior cerebral arteries (ACAs) were prominent (Supplementary Fig. 3).

Digital subtraction angiography (DSA) performed 3 days after the onset of symptoms demonstrated long, irregular stenosis of the left MCA with distal collateral branch runoff (Fig. 2A). The anterior territory of the left MCA was supplied by the leptomeningeal collaterals from the ipsilateral ACA (Fig. 2B); however, the anterior watershed region had scanty collaterals (Fig. 2B) with insufficient blood supply in the ipsilateral posterior cerebral artery (PCA) (Fig. 2C). Right carotid angiography revealed a steno-occlusive lesion with subsequent Moyamoya-like collateral branches (Fig. 2D). The right high frontal cortex was supplied by collaterals from the ipsilateral ACA and the anterior communicating artery (Fig. 2A, D); however, the posterior MCA territory had insufficient blood supply (Fig. 2E).

The patient was managed with atorvastatin $80 \mathrm{mg} /$ day and nimodipine $90 \mathrm{mg} /$ day according to the principles of SAH management, followed by concurrent administration of cilostazol $200 \mathrm{mg} /$ day after 5 days. His blood pressure stabilized at $140 / 90 \mathrm{mmHg}$. Follow-up brain CT performed 11 days after onset demonstrated near-complete resolution of cSAH compared with the baseline CT. Remarkably, the patient did not experience recurrent vascular events and was prescribed with cilostazol $200 \mathrm{mg}$, aspirin $100 \mathrm{mg}$, and atorvastatin $40 \mathrm{mg}$ to induce vasodilation and prevent secondary stroke. Follow-up imaging was performed after 10 months. MR contrast angiography revealed findings that were similar to those of the baseline TOF MR angiography. Perfusion-weighted imaging revealed mild delays in time to peak (TTP) in the right MCA territory with preserved blood flow and volume, whereas the left MCA territory showed normal findings (Fig. 2F). Written informed consent was obtained from the case patient regarding information on demographic data, medical condition, neuroimaging, and treatment.

\section{DISCUSSION}

In this patient, the possibility of reversible cerebral vasoconstriction syndrome was excluded because the patient had no history of vasoactive substance use, and DSA showed absence of alternating areas of stenosis and dilatation. Further, there was no evidence of Moyamoya disease characterized by a puff of smoke appearance, dural sinus thrombosis, or vascular malformation on DSA. MR imaging demonstrated neither typical findings of bilateral predominantly posterior cerebral edema of vasogenic origin nor evidence of multiple lobar, cortical, or subcortical hemorrhage.

The exact mechanism of cSAH associated with highgrade intracranial stenosis remains unknown in our patient; however, rupture of the leptomeningeal collaterals might have resulted in the development of the condition. More specifically, in cases of severe MCA

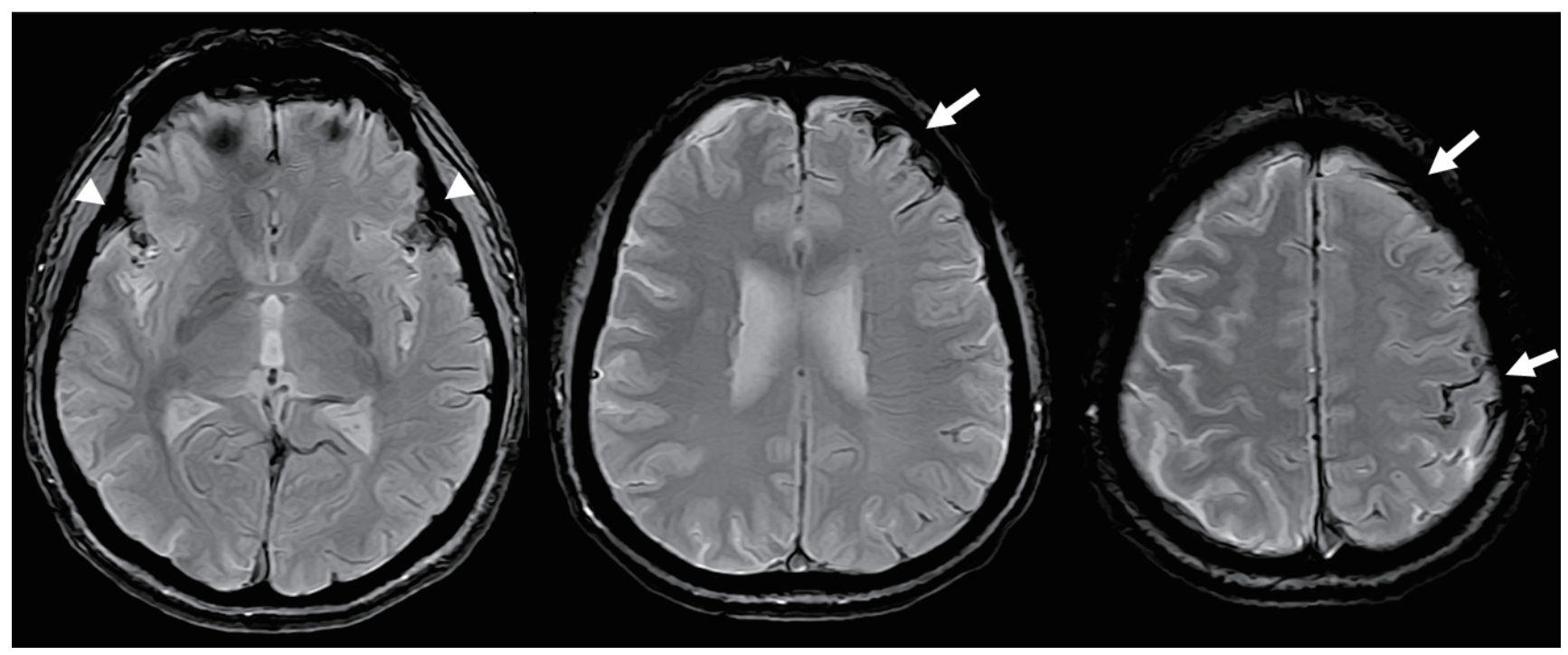

FIG. 1. Gradient-echo image shows hemorrhage along the left frontoparietal cortical sulci (arrows) and in the bilateral sylvian fissures (arrowheads). 
stenosis, dilated pial vessels (Fig. 2) that correlate with $\mathrm{cSAH}$ lesion may be prone to rupture due to an acute increase in hemodynamic stress. On the contrary, the tiny embolic infarcts in the left temporal lobe may have resulted from the Mi stenotic thrombus due to an increase in the perfusion pressure. The cSAH lesion in this patient was mainly located in the anterior (ACAMCA) and posterior (MCA-PCA) watershed regions. This finding is in line with those of previous studies, which indicated that cSAH resulting from hemodynamic stress develops in the watershed region. ${ }^{1-3}$ Our findings revealed that T'TP in the left anterior and posterior watershed regions were stationary, which indicat- ed the dilated status of the leptomeningeal vasculature to maintain blood flow.

High-grade stenosis was not clearly distinguished from non-atherosclerotic lesions. We failed to perform laboratory tests to assess for vasculitis due to patient's financial constraints. High-resolution MR imaging was not available to be performed in our hospital.

Our findings support the use of DSA in the diagnostic workup of patients with cSAH. ${ }^{4}$ However, further studies are warranted to determine whether DSA could be a useful diagnostic tool to assess the hemodynamically insufficient areas associated with cSAH in patients with significant atherosclerotic stenosis.
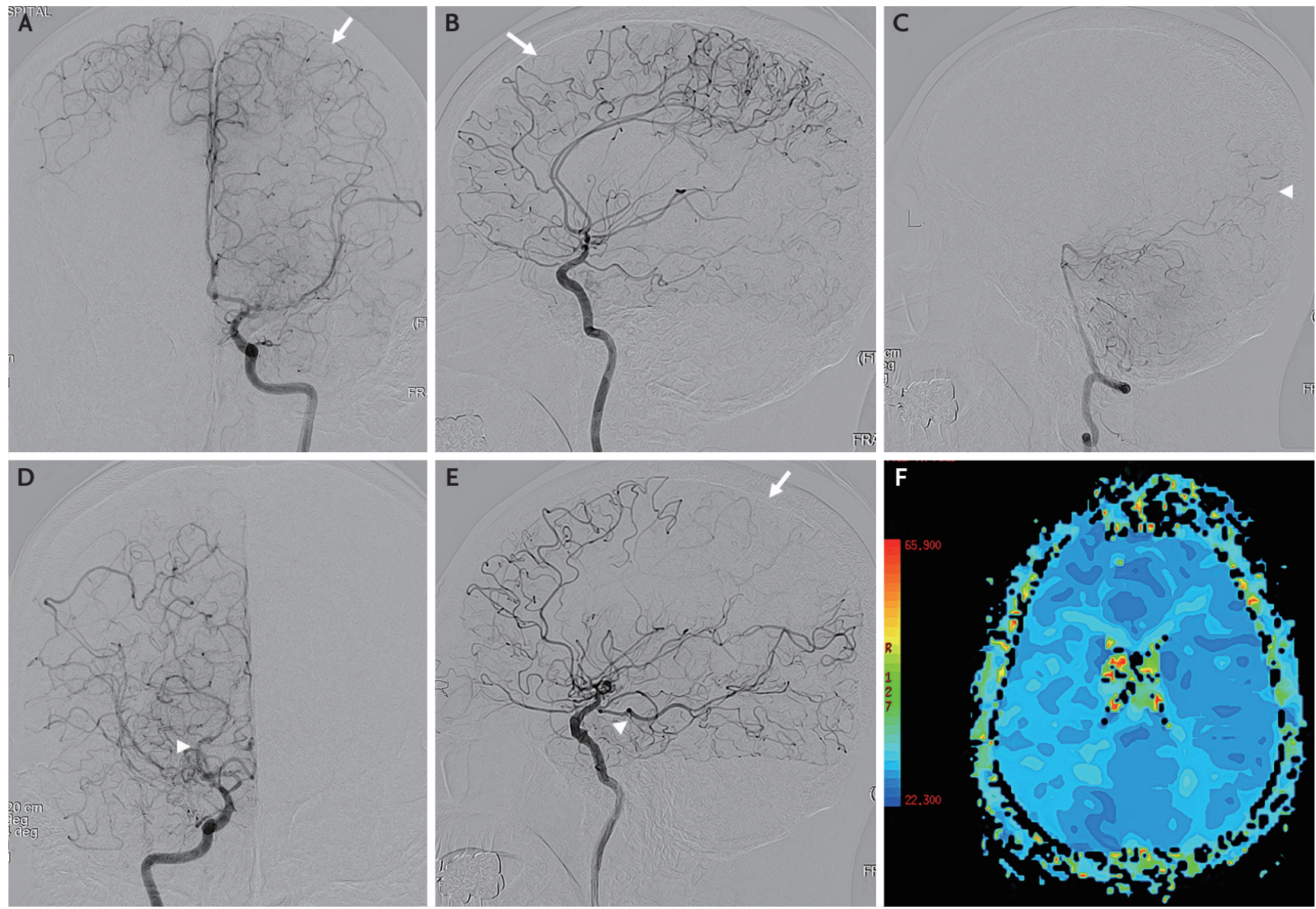

FIG. 2. Digital subtraction angiography of the (A, B) left internal carotid artery (ICA), (C) vertebral artery, and (D, E) right ICA, and (F) perfusion-weighted imaging. (A-C) The left middle cerebral artery (MCA) shows severe, long, irregular stenosis with distal leptomeningeal collaterals, whereas the ipsilateral anterior cerebral artery (ACA) shows prominent leptomeningeal collaterals bilaterally. The anterior watershed (arrow) of the left MCA and the posterior cerebral artery territory shows insufficient blood flow. (D, E) Steno-occlusive lesion of the right MCA with Moyamoya-like distal runoff. The right anterior hemisphere is supplied by the ipsilateral ACA and the anterior communicating artery (see also A). The right lateral view reveals relatively insufficient blood flow in the posterior aspect of the right MCA territory (arrow). The right posterior hemisphere is supplied by fetal circulation (arrowhead). (F) Perfusion-weighted image shows preserved time to peak, blood flow, and blood volume in the left MCA territory, whereas the time to peak of the right MCA territory is mildly delayed. 


\section{SUPPLEMENTARY MATERIALS}

Supplementary materials related to this article can be found online at https://doi.org/10.31728/jnn.2021.00099.

\section{Conflicts of Interest}

No potential conflicts of interest relevant to this article was reported.

\section{REFERENCES}

1. Geraldes R, Sousa PR, Fonseca AC, Falcão F, Canhão P, Pin- ho e Melo T. Nontraumatic convexity subarachnoid hemorrhage: different etiologies and outcomes. J Stroke Cerebrovasc Dis. 2014;23:е23-езо.

2. Chandra RV, Leslie-Mazwi TM, Oh D, Mehta B, Yoo AJ. Extracranial internal carotid artery stenosis as a cause of cortical subarachnoid hemorrhage. AJNR Am J Neuroradiol. 2011;32:E51-E52; author reply E53.

3. Kleinig TJ, Kimber TE, Thompson PD. Convexity subarachnoid haemorrhage associated with bilateral internal carotid artery stenoses. J Neurol. 2009;256:669-671.

4. Spitzer C, Mull M, Rohde V, Kosinski CM. Non-traumatic cortical subarachnoid haemorrhage: diagnostic work-up and aetiological background. Neuroradiology. 2005;47:525-531. 